\section{The hunting of the snark: the elusive calcium receptor(s)}

\author{
Lawrence G. Raisz \\ Department of Medicine, University of Connecticut Health Center, \\ Farmington, Connecticut, USA \\ J. Clin. Invest. 111:945-947 (2003). doi:10.1172/JCI200318235.
}

"For the snark's a peculiar creature that won't be canght in a commonplace way." -Lewis Carroll, The Hunting of the Snark

The first hunt took a long time. The existence of a highly sensitive calciumsensing receptor (CaSR) was postulated more than 30 years ago based on the exquisite ability of the parathyroid cell to respond to small changes in serum ionized calcium concentration (1). The gene encoding CaSR was finally cloned in 1993 (2). Shortly thereafter it was found that inactivating mutations in CaSR were responsible for familial hypocalciuric hypercalcemia $(\mathrm{FHH})$ in heterozygotes and for severe hyperparathyroidism in homozygous infants while activating mutations caused hypoparathyroidism $(3,4)$. Deletion of the mouse calcium receptor mimics the phenotype of FHH in heterozygote individuals. In homozygous mice there is early death due to hypercalcemia and a surprising skeletal phenotype of rickets, which suggested that CaSR might be essential for normal mineralization $(5,6)$.

\section{Is CaSR important in bone?}

There is substantial evidence that the classical Ca receptor also plays a role in the regulation of calcitonin secre-

\footnotetext{
Address correspondence to: Lawrence G. Raisz, University of Connecticut Health Center, Department of Medicine, General Clinical Research Center, MC3805,

263 Farmington Avenue, Farmington,

Connecticut 06032, USA.

Phone: (860) 679-3851; Fax: (860) 679-3209;

E-mail: raisz@nso.uchc.edu.

Conflict of interest: The author has declared that no conflict of interest exists.

Nonstandard abbreviations used: calciumsensing receptor (CaSR); familial hypocalciuric hypercalcemia $(\mathrm{FHH})$; glial cells missing 2 (Gcm2); parathyroid hormone (PTH).
}

High concentrations of calcium can also inhibit osteoclast activity (12). These responses differ from the parathyroid response not only in requiring large changes in calcium concentration, but also in their ion selectivity (13).

\section{Double knockouts rescue the mice and their bones}

In this issue of the JCI, two groups of investigators have developed ingenious double knockout models to permit CaSR-deficient mice to survive in an effort to define the potential importance of CaSR in mouse tissues other than the parathyroid glands. In the study by Kos et al. (14) the phenotype was rescued from hyperparathyroidism by the simple and direct approach of genetic ablation of the PTH gene itself. Quite a different strategy was used by Tu et al. (15). Previous studies had shown that deletion of the glial cells missing 2 (Gcm2) gene in the mouse resulted in failure of the development of the parathyroid glands (16). These animals exhibited only mild hypoparathyroidism. Both of these studies remind us that gene deletion may be a good way to "hunt for snarks". The study by $\mathrm{Tu}$ et al. uncovered an auxiliary site for the regulation of calcium homeostasis: PTH production in the thymus. The importance of the role of this thymus source in humans is uncertain, since patients who have had all four parathyroids removed generally have severe hypoparathyroidism. The parathyroid hyperplasia that was observed by Kos induced cAMP in response to high cal- cium concentrations (11).

tion in the thyroid (7) and ion transum, but also, probably indirectly, for potassium (8). The receptor has also including bone cells. However the role of this highly sensitive receptor in the largest role in controlling calcium in the body, remains elusive (9). Bone required to produce cellular effects are much larger than the $1-2 \%$ changes in roid hormone secretion (PTH) secretion osteoblastic cells, high co replication and differentiation and the expression of the inducible cyclooxygenase COX-2 (10). Howeve from $C a s R$ gene-knockout mice show a stimulation of DNA synthesis, activation of a serum response element and inhibition of agonist-
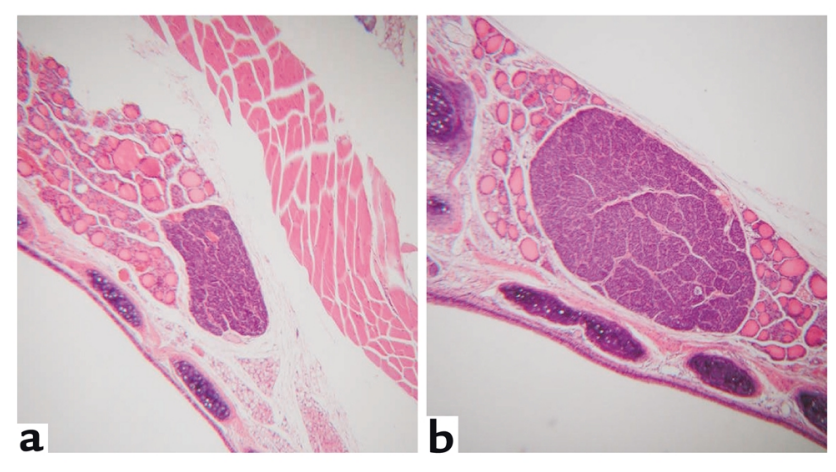

Figure 1

Upon comparison with sex-matched control littermates (a) $\mathrm{PTH}^{-1-} \mathrm{CaSR} \mathrm{R}^{-1-}$ mice exhibited parathyroid hyperplasia (b) (14). The enlargement of the parathyroid glands in $\mathrm{PTH}^{-1-} \mathrm{CaSR}^{-/-}$animals could indicate that CaSR plays an important regulatory role in limiting parathyroid size and cell replication. 
a
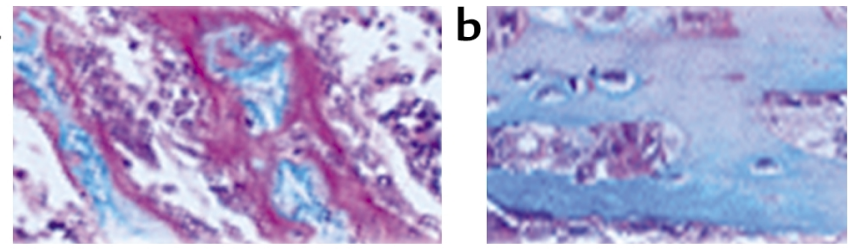

Figure 2

Nondecalcified histologic sections of the tibia of 1-week-old (a) CasR-deficient, and (b) CasR-and $\mathrm{Gcm} 2$-deficient mice. Double knockout reverses the mineralization defect observed in Cas $R^{-/-}$mice. This result indicates that an excess of PTH may somehow directly produce a picture of rickets and osteomalacia and that this abnormality is not due to the absence of CaSR in bone cells. It will be important to find out what very high PTH levels might be doing to the proteins and transport systems that regulate mineralization in these mice.

et al. may be the signal for another snark hunt (Figure 1). The results imply that CaSR regulates parathyroid cell replication independently of PTH secretion. The development of primary hyperparathyroidism in transgenic mice over-expressing cyclin D1 (17) suggests the existence of such a pathway and it will be important to find out how CaSR might affect this pathway.

The results of these two studies were concordant and perhaps disappointing in that both forms of rescue resulted in the production of mice with an essentially normal skeletal phenotype. However, there were abnormalities of calcium regulation. In the Gcm2-deficient mice hypocalciuria persisted while in the PTHknockout animals there was increased expression of calcium transporters in the kidney and greater variability of serum calcium concentration and of urine calcium excretion. This is presumably due to absence of fine regulation by PTH.

Perhaps disappointing was the finding that the skeletal changes associated with CaSR deficiency were essentially abrogated in both models. The mice rescued by Gcm2 deficiency showed no detectable skeletal abnormalities while there was a small increase in bone mineral density of vertebrae of the female $\mathrm{PTH}^{-/}$rescued animals but no significant changes by dynamic histomorphometry. However, neither model was subjected to perturbations of skeletal remodeling, which might bring out a role for CaSR. On the other hand, the results suggest that the severe osteomalacia seen in single knockout (Figure 2) is a direct consequence of PTH excess, and this raises new questions.

\section{Where is the snark?}

How can we now reconcile the data demonstrating that calcium regulates bone cell function, as well as the function of many other cells, with the finding that CaSR-deficient mice show so few abnormalities when hyperparathyroidism is prevented? There is evidence for calcium responsiveness in bone cells that lack CaSR. This should be no surprise since calcium regulation of cell function is so fundamental in biology and since the concentration-response relations for calcium effects as well as ferent tissues. On the other hand there is evidence for Cas $R$ gene expression in bone. Could the present results indicate that this expression is vestigial or nonfunctional and that other calcium receptors in cells are even more important than CaSR? For example, there are glutamate receptors present in bone that may play a role in the response to mechanical forces (18), and these receptors are responsive to large changes in the calcium concentration (19).

To resolve these questions it will be important to determine whether CaSR is truly a regulator in bone. This will require demonstration that the functional protein is present in the cell membrane. On the other hand, a careful analysis of cellular responses to calcium in cells lacking CaSR should also be possible using the present models, leading to identification of other elusive calcium receptors. Inducible and/or targeted knockout of CasR in bone cells may be necessary to fully define its function since embryonic knockout may produce compensatory pathways. The alternative calcium-sensing mechanisms that regulate cell function in the absence of CaSR might involve other classical the ion selectivity appear to differ in dif- transmembrane proteins, but might also involve alterations in membrane function through interaction of calcium with membrane phospholipids. Whatever the mechanism, the effects of calcium are likely to be critically important in physiologic and pathologic regulation of bone cells. This could be true not only for high calcium concentrations, which might accelerate bone formation and decrease bone resorption, but for low concentrations that might have the opposite effect. The effect of lowering calcium concentration is striking in the parathyroid gland, but has not been examined extensively in bone cells. If phosphate concentration is increased locally during mineralization, calcium concentration is likely to decrease. This might be the signal that turns off further matrix synthesis by osteoblasts. The Neumans pointed out more than 50 years ago that extracellular fluid is super-saturated relative to the bone mineral, hydroxyapatite (20). Neuman and Neuman postulated that the removal of a cell and/or protein coating of the mineral would result in a local drop in calcium concentration that could enhance osteoclast migration and activity. We must learn more about how bone cells sense calcium to determine how this all-important ion that is stored in bone can regulate its own fate.

1. Brown, E.M. 1991. Extracellular Ca2+ sensing, regulation of parathyroid cell function, and role of $\mathrm{Ca} 2+$ and other ions as extracellular (first) messengers. Physiol. Rev. 71:371-411.

2. Brown, E.M., et al. 1993. Cloning and characterization of an extracellular $\mathrm{Ca}(2+)$-sensing receptor from bovine parathyroid. Nature. 366:575-580.

3. Pollak, M.R., et al. 1993. Mutations in the human $\mathrm{Ca}(2+)$-sensing receptor gene cause familial hypocalciuric hypercalcemia and neonatal severe hyperparathyroidism. Cell. 75:1297-1303.

4. Pollak, M.R., Seidman, C.E., Brown, E.M. 1996 Three inherited disorders of calcium sensing. Medicine. 75:115-123.

5. Ho, C., et al. 1995. A mouse model of human familial hypocalciuric hypercalcemia and neonatal severe hyperparathyroidism. Nat. Genet. 11:389-394

6. Garner, S.C., Pi, M., Tu, Q., and Quarles, L.D. 2001. Rickets in cation-sensing receptor-deficient mice: an unexpected skeletal phenotype. Endocrinology. 142:3996-4005.

7. Garrett, J.E., et al. 1995. Calcitonin-secreting cells of the thyroid express an extracellular calcium receptor gene. Endocrinology. 136:5202-5211.

8. Watanabe, S., et al. 2002. Association between activating mutations of calcium-sensing receptor and Bartter's syndrome. Lancet. 360:692-694.

9. Quarles, L.D. 1997. Cation sensing receptors in bone: a novel paradigm for regulating bone remodeling? J. Bone Miner. Res. 12:1971-1974.

10. Choudhary, S., et al. 2001. Extracellular calcium transcriptionally induces cyclooxygenase- 2 in 
osteoblasts via an ERK signaling pathway. J. Bone Miner. Res. 16:s427.

11. Pi, M., Garner, S.C., Flannery, P., Spurney, R.F., and Quarles, L.D. 2000. Sensing of extracellular cations in CasR-deficient osteoblasts. Evidence for a novel cation-sensing mechanism. J. Biol. Chem. 275:3256-3263.

12. Kanatani, M., Sugimoto, T., Kanzawa, M., Yano, S., and Chihara, K. 1999. High extracellular calcium inhibits osteoclast-like cell formation by directly acting on the calcium-sensing receptor existing in osteoclast precursor cells. Biochem. Biophys. Res. Commun. 261:144-148.

13. Zaidi, M., Adebanjo, O.A., Moonga, B.S., Sun,
L., and Huang, C.L. 1999. Emerging insights into the role of calcium ions in osteoclast regulation. J. Bone Miner. Res. 14:669-674.

14. Kos, C.H., et al. 2003. The calcium-sensing receptor is required for normal calcium homeostasis independent of parathyroid hormone. J. Clin. Invest. 111:1021-1028. doi:10.1172/JCI200317416.

15. Tu, Q., et al. 2003. Rescue of the skeletal phenotype in CasR-deficient mice by transfer onto the Gcm 2 null background. J. Clin. Invest. 111:1029-1037. doi:10.1172/JCI200317054. 16. Gunther, T., et al. 2000. Genetic ablation of parathyroid glands reveals another source of parathyroid hormone. Nature. 406:199-203. 17. Imanishi, Y., et al. 2001. Primary hyperparathy- roidism caused by parathyroid-targeted d over expression of cyclin D1 in transgenic mice. J. Clin. Invest. 107:1093-1102.

18. Skerry, T.M. 1999. Identification of novel signaling pathways during functional adaptation of the skeleton to mechanical loading: the role of glutamate as a paracrine signaling agent in the skeleton. J. Bone Miner. Metab. 17:66-70.

19. Kubo, Y., Miyashita, T., and Murata, Y. 1998 Structural basis for a Ca2+-sensing function of the metabotropic glutamate receptors. Science. 279:1722-1725.

20. Neuman, W., and Neuman, M.W. 1958. The chemical dynamics of bone mineral. University of Chicago Press. Chicago, Illinois, USA. 209 pp.

\title{
Negative regulators of sodium transport in the kidney: Key factors in understanding salt-sensitive hypertension?
}

\author{
Bernard C. Rossier \\ Institut de Pharmacologie et de Toxicologie, Université de Lausanne, Lausanne, Switzerland \\ J. Clin. Invest. 111:947-950 (2003). doi:10.1172/JCI200318232.
}

\section{Genetic evidence supporting Guyton's hypothesis: the kidney is a main player in the long-term control of blood pressure}

Hypertension is the most common disease of the human population. Both genetic and non-genetic factors are involved and high salt intake has been proposed as a major risk factor. According to the hypothesis put forward by Guyton, over 20 years ago, control of blood pressure in the steady-state and on a long-term basis is critically dependent on renal mechanisms (1). During the last decade, a number of genes expressed in the distal part of the nephron have been shown to be directly involved in the control of blood pressure (2). Muta-

\footnotetext{
Address correspondence to: Bernard C. Rossier, Institut de Pharmacologie et de Toxicologie, Université de Lausanne, Rue du Bugnon 27, CH-1005 Lausanne, Switzerland. Phone: 41-21-692-535; Fax: 41-21-692-5355; E-mail: Bernard.Rossier@ipharm.unil.ch. Conflict of interest: The author has declared that no conflict of interest exists.

Nonstandard abbreviations used: pseudohypoaldosteronism type II (PHAII); with no lysine $\mathrm{K}$ (WNK); $\mathrm{Na}-\mathrm{Cl}$ cotransporter (NCC); epithelial sodium channel (ENaC).
}

tions in single genes that cause isolated Mendelian syndromes characterized by hypertension or hypotension have been identified and, remarkably, these genes have all turned out to be involved in the control of sodium chloride absorption by the distal nephron (Table 1). The distal nephron (Figure 1) is composed of the distal convoluted tubule, the connecting tubule and various segments of the collecting duct. The fact that these are the terminal segments of the nephron affords them the final word, so to speak, on how much salt is excreted. This underlies their critical importance in the maintenance of salt balance, which in turn controls the volume of the extracellular space and blood pressure. The reabsorption of sodium in the distal tubule and the collecting tubules is closely regulated, chiefly by the action of the hormone aldosterone.

\section{WNK kinases repress $\mathrm{Na}-\mathrm{Cl}$ cotransporter activity}

Pseudohypoaldosteronism type II (PHAII) or Gordon syndrome is an autosomal dominant disease, characterized by hypertension and hyper- kalemia, despite a normal glomerular filtration rate. In addition, plasma renin levels are low, however plasma aldosterone is often in the normal range, but inappropriately low with respect to the observed high level of plasma potassium, a strong stimulus of aldosterone secretion $(3,4)$. Diseaseassociated physiological abnormalities can be corrected by administration of thiazide diuretics. The use of the term pseudohypoaldosteronism here is somewhat questionable since salt retention is observed in this syndrome. Hypertension, rather than salt wasting, is also observed. We prefer the term familial hyperkalemic hypertension, which more accurately describes the pathophysiology. The disease is genetically heterogeneous and three loci have been identified on chromosomes 1 (PHA2A), 17 (PHA2B), and 12 (PHA2C). Furthermore, genetic heterogeneity demonstrate the existence of at least a fourth locus (3). Mutations in two members of a novel serine/threonine kinase family named WNK (with no lysine $\underline{\mathrm{K}}$, i.e. lacking a lysine typical of the catalytic domain of this kinase family) - WNK1 and WNK4 - map to the PHA2A and PHA2B loci, respectively. The WNK1 mutations are large intronic deletions that were postulated to increase gene expression and increase enzymatic activity (4). In contrast, WNK 4 mutations $(\mathrm{E} 562 \mathrm{~K}, \mathrm{D} 564 \mathrm{~A}$, and Q565E) map to a small stretch of amino acids that lies outside the catalytic domain that is fully conserved between WNK1 and WNK4 (4). WNK1 is ubiquitously expressed in many tissues and organs and seems restricted to diverse chloride-transporting epithelia (5). In the kidney, WNK1 is restricted to the aldosterone-sensitive distal nephron 